\title{
Benzophenone Ultrafast Triplet Population: Revisiting the Kinetic Model by Surface-Hopping Dynamics
}

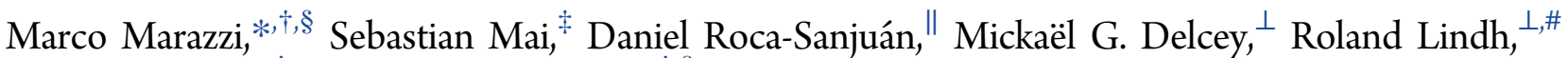 \\ Leticia González, ${ }^{\star}$ and Antonio Monari* ${ }^{\dagger}, \S$ \\ ${ }_{\dagger}^{\dagger}$ Théorie-Modélisation-Simulation, Université de Lorraine - Nancy, SRSMC, Boulevard des Aiguillettes, 54000 \\ Vandoeuvre-lès-Nancy, Nancy, France

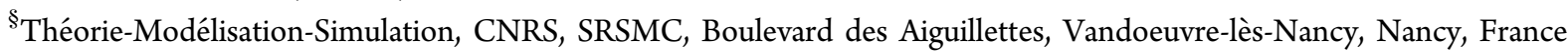 \\ ${ }^{\ddagger}$ Institute of Theoretical Chemistry, University of Vienna, Währinger Straße 17, A-1090 Vienna, Austria \\ "Instituto de Ciencia Molecular, Universitat de València, P.O. Box 22085, ES-46071 València, Spain

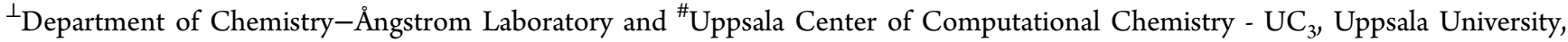 \\ Uppsala 75105 , Sweden
}

\section{Supporting Information}

ABSTRACT: The photochemistry of benzophenone, a paradigmatic organic molecule for photosensitization, was investigated by means of surface-hopping ab initio molecular dynamics. Different mechanisms were found to be relevant within the first $600 \mathrm{fs}$ after excitation; the longdebated direct $\left(S_{1} \rightarrow T_{1}\right)$ and indirect $\left(S_{1} \rightarrow T_{2} \rightarrow T_{1}\right)$ mechanisms for population of the low-lying triplet state are both possible, with the latter being prevalent. Moreover, we established the existence of a kinetic equilibrium between the two triplet states, never observed before. This fact implies that a significant fraction of the overall population resides in $\mathrm{T}_{2}$, eventually allowing one to revisit the usual spectroscopic assignment proposed by transient absorption spectroscopy. This finding is of particular interest for photocatalysis as well as for DNA damages studies because both $\mathrm{T}_{1}$ and $\mathrm{T}_{2}$ channels are,

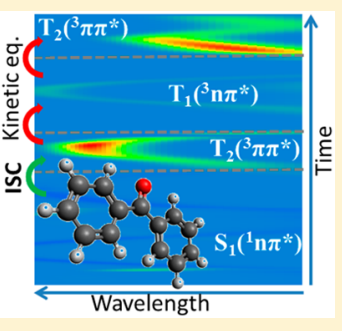
in principle, available for benzophenone-mediated photoinduced energy transfer toward DNA.

$\mathrm{F}$ ast intersystem crossing (ISC) mechanisms occurring in organic molecules have been extensively studied in the last decades, especially by time-resolved experimental techniques. $^{1-8}$ Nevertheless, it is only in the last years that reliable computational methods were developed in order to study the dynamical details of such processes. ${ }^{9-13}$ A comparison between experimental and theoretical results can be of particular interest in the case of aromatic ketones because different analyses of diverse experiments can lead to contradictory results. In this regard, a paradigmatic case is benzophenone (BP), a wellknown building block in organic synthesis, recently becoming more and more interesting as a crucial agent in different applications. Indeed, thanks to its ability to efficiently absorb ultraviolet (UV) light, it may be used as a photoinitiator in phototherapeutic applications, ${ }^{14}$ as a UV-blocker to prevent photodegradation in polymers ${ }^{15}$ and soaps, ${ }^{16}$ as a photophysical probe to clarify peptide-peptide interactions, ${ }^{17}$ or as a photosensitizer to transfer energy to DNA. ${ }^{18,19}$ Given the wide applicability of BP in so many different fields (from materials science and technology to biology and pharmacology), a clear-cut insight into the mechanisms undergoing the initial photophysical and photochemical processes is necessary.

A number of questions are still open from previous experimental studies. After population of the lowest-lying singlet excited state $S_{1}$ - either by absorption in the UVA $\left(S_{0}\right.$ $\rightarrow S_{1}$ ) or after an ultrafast radiationless internal conversion from the optically brighter $S_{2}$ state $\left(S_{0} \rightarrow S_{2} \rightarrow S_{1}\right)$-two pathways have been postulated to populate the lowest-lying triplet state via a spin-orbit coupling (SOC) mechanism: direct $\left(\mathrm{S}_{1} \rightarrow \mathrm{T}_{1}\right)^{1,3,4}$ or indirect $\left(\mathrm{S}_{1} \rightarrow \mathrm{T}_{2} \rightarrow \mathrm{T}_{1}\right)^{2,6-8}$ This unresolved aspect leaves open questions concerning BP emission properties. Indeed, according to the previous mechanisms, only phosphorescence from the $T_{1}$ minimum would be, in principle, expected. However, gas-phase experiments proposed a long-living emission following a biexponential decay, suggesting that dual phosphorescence could occur from both an unrelaxed and a vibrationally relaxed triplet state. ${ }^{5}$ Additionally, fluorescence was also observed as an E (eosin)type delayed event ${ }^{20}$ (see Scheme 1).

At present, only one computational mechanistic study was reported on the photochemistry of $\mathrm{BP}$, based on accurate (CASPT2//CASSCF) calculations of minimum energy paths (MEPs) and crossing points between potential energy surfaces (PESs). ${ }^{21}$ The results point toward an indirect population of the $\mathrm{T}_{1}$ state via the intermediate $\mathrm{T}_{2}$. Additionally, an $S_{1}$ minimum was located, which could explain delayed fluorescence.

Here, we investigate BP photochemistry and photophysics from a different and complementary perspective, namely, directly taking into account the time evolution of the different

Received: December 16, 2015

Accepted: January 28, 2016

Published: January 28, 2016 
Scheme 1. (a) Obtained Characteristic BP Coordinates at Franck-Condon (CASSCF(12,11)/ANO-S-VDZP level of theory) ${ }^{a}$ and (b) Possible Photochemical Mechanisms Involving BP, Including Photon Absorption ( $\left.h \nu_{\mathrm{abs}}\right)$, Fluorescence $\left(h \nu_{\text {fluo }}\right)$, and Phosphorescence $\left(h \nu_{\mathrm{ph}}\right)$ from the Respective PES Minima $\left(\min ^{b}\right)$

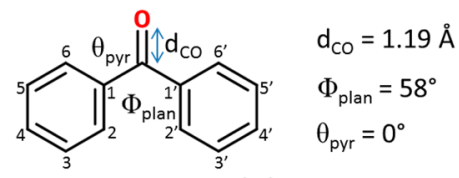

(a)

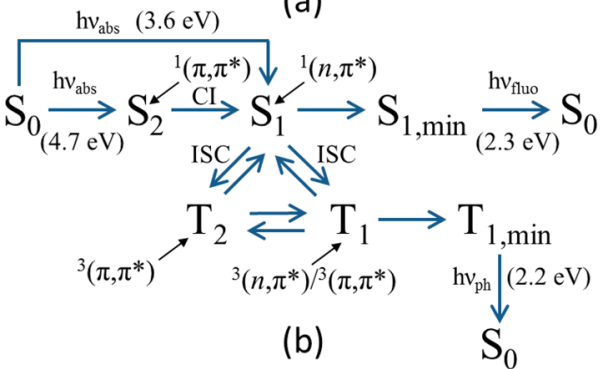

${ }^{a}$ The $\mathrm{C}=\mathrm{O}$ distance $\left(d_{\mathrm{CO}}\right)$ and improper dihedrals that measure the angle between phenyl planes $\left(\Phi_{\text {plan }}\right.$ : $\left.\mathrm{C} 2-\mathrm{C} 1-\mathrm{C}^{\prime}-\mathrm{C} 2^{\prime}\right)$ and carbonyl carbon pyramidalization $\left(\theta_{\mathrm{pyr}}: \mathrm{C}=\mathrm{O}-\mathrm{C} 1-\mathrm{Cl}^{\prime}\right)$. Their evolution in time is shown in the Supporting Information. ${ }^{b}$ The energy values are taken from the literature and correspond to the CASPT2 $(12,11)$ / ANO-L-VDZP//CASSCF(12,11)/ANO-L-VDZP level of theory. ${ }^{21}$ The electronic nature of the states is specified. CI: conical intersection; ISC: intersystem crossing.

excited states. To this aim, we used CASSCF-based nonadiabatic molecular dynamics including surface-hopping ${ }^{22}$ between electronic adiabatic states of same and different multiplicity, on the same footing. The CASSCF $(12,11) /$ ANOS-VDZP level of theory was selected for its qualitative agreement with more accurate CASPT2(12,11)/ANO-LVDZP calculations while still being computationally efficient for dynamics. ${ }^{21}$ The selected active space includes two pairs of $\pi \pi^{*}$ orbitals for each phenyl ring, a $\pi \pi^{*}$ pair of orbitals centered on the carbonyl group and an $\mathrm{n}$ orbital of the oxygen atom. When including water as the solvent with the implicit polarizable continuum model, ${ }^{23}$ the same qualitative and semiquantitative optical properties were found for the most representative BP structures (see the Supporting Information). Hence, dynamics simulations were performed only in the gas phase.

Although, as previously stated, diverse unresolved questions need to be answered, there is agreement between theory and experiment concerning the electronic absorption of the Franck-Condon (FC) structure; because $S_{1}$ and $S_{2}$ states are, respectively, ${ }^{1}\left(\mathrm{n}, \pi^{*}\right)$ and ${ }^{1}\left(\pi, \pi^{*}\right)$, the $S_{0} \rightarrow S_{2}$ electronic transition is more probable than the $S_{0} \rightarrow S_{1}$. Nevertheless, the first three low-lying triplet states are located below $S_{1}$. Therefore, a direct $\left(S_{0} \rightarrow S_{1}\right)$ or indirect $\left(S_{0} \rightarrow S_{2} \rightarrow S_{1}\right)$ population of the lowest-lying singlet excited state is a prerequisite for the subsequent intersystem crossing; at FC, $S_{1}$ is almost degenerate in energy with $T_{2}$ and $T_{3}(0.07 \mathrm{eV}$ at the CASPT2 level $\left.{ }^{21}\right)$, both ${ }^{3}\left(\pi, \pi^{*}\right)$ states. According to the ElSayed's rules, ${ }^{24}$ an ISC process governed by a direct SOC interaction is efficient when a change in the molecular orbital type occurs, hence in principle favoring an $S_{1} \rightarrow T_{2,3}$ (that is, $\left.{ }^{1}\left(\mathrm{n}, \pi^{*}\right) \rightarrow{ }^{3}\left(\pi, \pi^{*}\right)\right)$ SOC mechanism. On the other hand, the larger energy separation between $S_{1}$ and $T_{1}(0.53 \mathrm{eV}$ at the
CASPT2 level ${ }^{21}$ ) and the fact that $\mathrm{T}_{1}$ is a mixed ${ }^{3}\left(\mathrm{n}, \pi^{*}\right) /^{3}\left(\pi, \pi^{*}\right)$ state $^{21}$ due to the nonplanarity of the BP molecule (Scheme 1$)$ suggest that a direct $\left(S_{1} \rightarrow T_{1}\right)$ SOC mechanism should be disfavored but still possible. We note that we focus in this work on the ISC processes after excitation to the $S_{1}$ state. While there might be relevant ISC pathways starting in $S_{2}$, this state is located at much higher excitation energies, which we do not consider here. This can also be evidenced from a recent experimental study. ${ }^{25}$ Moreover, the $S_{1}$ state is relevant in DNA photosensitization. Indeed, it appears at lower energies than the lowest-lying states of the nucleobases and can transfer its triplet energy to the triplet of thymine. ${ }^{19}$

The gas-phase nonadiabatic dynamics simulations were started on a set of 65 initial conditions. To this end, the ground-state PES was locally reproduced by a frequency calculation at the equilibrium geometry, that is, by a harmonic approximation, and phase space points were sampled from the Wigner distribution. Assuming a vertical $S_{0} \rightarrow S_{1}$ transition, all trajectories were run for a maximum of $600 \mathrm{fs}$, considering a stable triplet population as a criterion to stop the simulation (see the Supporting Information). All calculations and further analyses were performed with the SHARC (Surface Hopping including Arbitrary Couplings) software suite ${ }^{13}$ coupled to MOLCAS $8,{ }^{26}$ including Cholesky decomposition for the calculation of the energy gradients. ${ }^{27,28}$

At $600 \mathrm{fs}, 60 \%$ of the trajectories reached the triplet manifold, as evidenced from Figure 1. Besides singlet-triplet

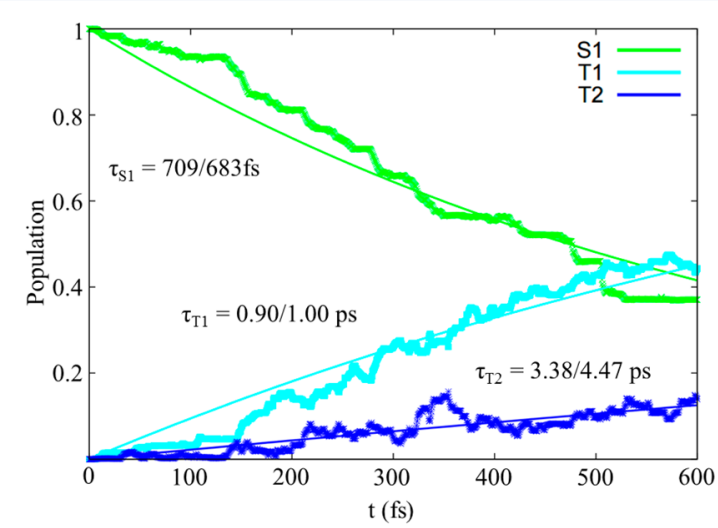

Figure 1. Kinetic models of the BP photoreactivity, based on global fitting to a single-exponential rate law. The $S_{1}$ population decays over the time, giving rise to $T_{1}$ and $T_{2}$ formation. The lifetime values $\tau$ are shown; the first (second) number corresponds to a parallel (serial) kinetic model. The two curves are nearly identical between 0 and 600 fs. Equations and curves above $600 \mathrm{fs}$ can be found in the Supporting Information.

interconversion, the kinetic model reveals that the $S_{1}$ exponential decay mainly results in the population of $\mathrm{T}_{1}$. Nevertheless, a population of $10 \%$ in $\mathrm{T}_{2}$ is also observed (within $600 \mathrm{fs}$ ), characterized by a distinctive characteristic formation time $\tau$ (ca. 4 times larger for $\mathrm{T}_{2}$, with respect to $\mathrm{T}_{1}$ ) and therefore cannot be neglected. $T_{3}$ population, on the other hand, is a nonrelevant event (see the Supporting Information). The persistent $T_{2}$ population is due to a kinetic equilibrium between $T_{1}$ and $T_{2}$ that governs the initial triplet manifold population, and it can play a role for $\mathrm{BP}$ ultrafast spectroscopic characterization, that is, before phosphorescence is observed. Experimentally, the time scale recorded for BP triplet formation is different depending on the environment. By picosecond 


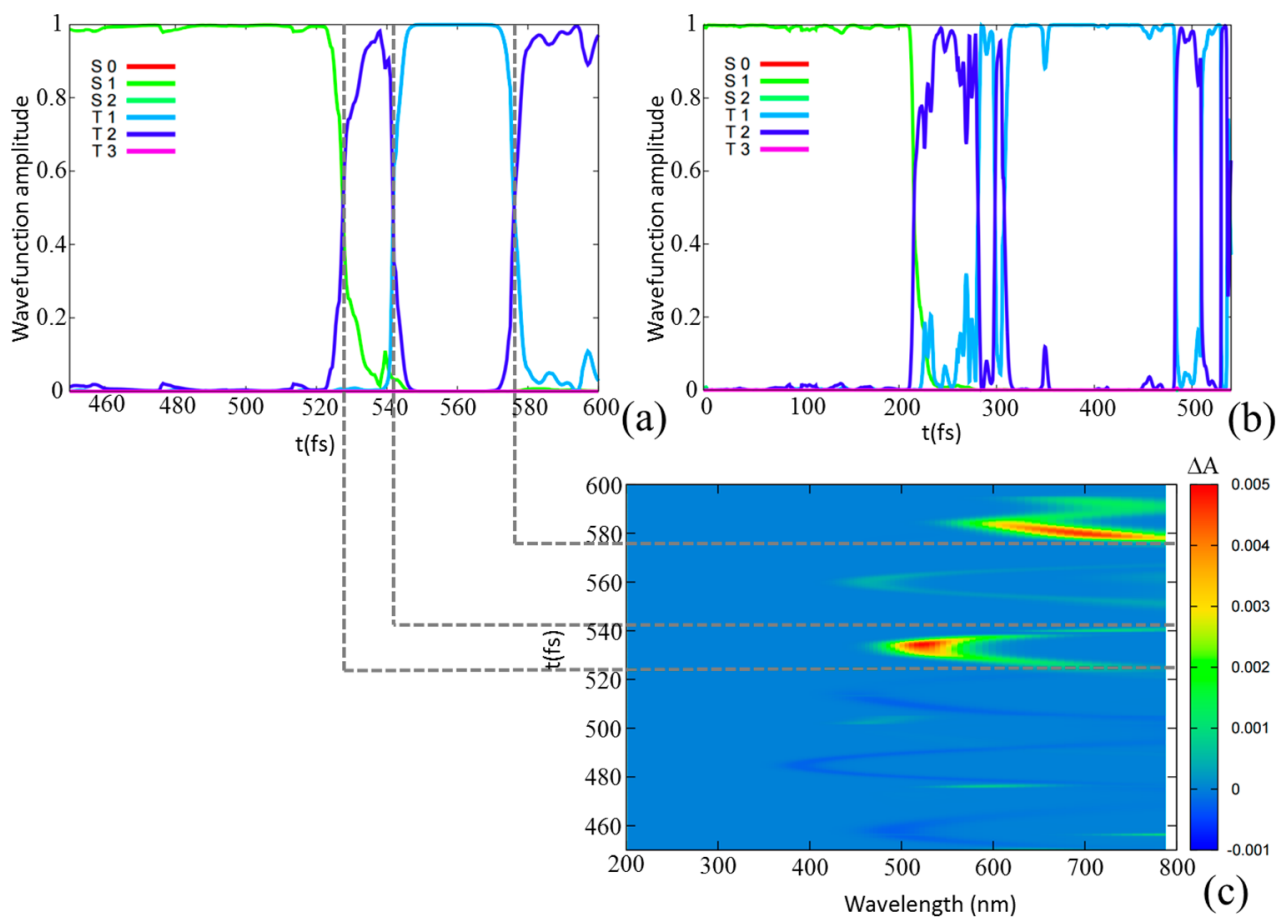

Figure 2. Representative excited-state trajectories showing an $\mathrm{S}_{1} \rightarrow \mathrm{T}_{2} \rightarrow \mathrm{T}_{1}$ indirect mechanism and the subsequent equilibrium between $\mathrm{T}_{1}$ and $\mathrm{T}_{2}$ states $(\mathrm{a}, \mathrm{b})$. The transient absorption $(\Delta A)$ spectrum is simulated for one of the trajectories (c). Positive $\Delta A$ values correspond to excited-state absorption; negative $\Delta A$ values indicate stimulated emission.

time-resolved spectroscopy, a global $\tau_{\text {ISC }} \approx 25$ ps was found for BP single crystals, ${ }^{8}$ which decreases in the liquid phase $\left(\tau_{\text {ISC }} \approx\right.$ 6.5-16 ps, depending on the solvent $\left.{ }^{7,29,30}\right)$ and even more in the gas phase. Indeed, the latter was determined by photoelectron spectroscopy that assigned a lifetime of $5 \pm$ $0.5 \mathrm{ps}$ to the formation of $\mathrm{T}_{1}$ as the latter is considered as the stable final state. ${ }^{25}$ Our exponential kinetic models predict comparable values (3.38 and $4.47 \mathrm{ps}$ ) for $\mathrm{T}_{2}$, while the predicted $\tau_{\mathrm{T} 1}(0.90$ and $1.00 \mathrm{ps})$ was not experimentally detected.

In detail, two kinetic models were considered: (i) parallel formation of $T_{1}$ and $T_{2}$ from $S_{1}$, congruent with our simulation results and (ii) serial formation of either $T_{1}$ or $T_{2}$ from $S_{1}$, in agreement with the usual experimental fitting procedure.

This apparent disagreement can be explained by the peculiarity of BP photophysics, as enlightened by our simulations. As can be seen in Figure $2 \mathrm{a}, \mathrm{b}$ (and in the Supporting Information), after $S_{0} \rightarrow S_{1}$ vertical excitation, the system evolves toward a region where $S_{1}, T_{1}$, and $T_{2}$ are almost degenerate in energy, while the $\mathrm{T}_{3}$ energy rises (in accordance with the CASPT2 $\mathrm{MEP}^{21}$ ). From the FC region to the ISC point, the dynamics is described exclusively by $S_{1}$ (i.e., $S_{2}$ is not populated), a ${ }^{1}\left(\mathrm{n}, \pi^{*}\right)$ state that shows mainly low-intensity stimulated $S_{1} \rightarrow S_{0}$ emission. Once the ISC point is reached, the triplet manifold is populated, and two signals $\left(\mathrm{T}_{1} \rightarrow \mathrm{T}_{3}\right.$ and $\mathrm{T}_{2} \rightarrow \mathrm{T}_{3}$ excited-state absorptions) could, in principle, be detected by transient spectroscopy since, because of the quasidegeneracy, $\mathrm{T}_{1} \rightarrow \mathrm{T}_{2}$ absorption would have a much too low excitation energy. Electronically, the $\mathrm{T}_{1}$ corresponds to a mixed ${ }^{3}\left(\mathrm{n}, \pi^{*}\right) /^{3}\left(\pi, \pi^{*}\right)$ state, while $\mathrm{T}_{2}$ and $\mathrm{T}_{3}$ are ${ }^{3}\left(\pi, \pi^{*}\right)$ states; therefore, the $\mathrm{T}_{2} \rightarrow \mathrm{T}_{3}$ transition will have a significantly higher oscillator strength. Furthermore, because of $T_{1}$ and $T_{2}$ degeneracy, the two transitions will happen in the same spectral region, and hence, the brighter $T_{2} \rightarrow T_{3}$ transition will totally mask the low $\mathrm{T}_{1} \rightarrow \mathrm{T}_{3}$ intensity. This can be evidently evidenced from Figure $2 c$, where we reported the inhomogeneous $^{31}$ time-dependent spectrum simulated for only one trajectory (see the Supporting Information); the excited-state absorption reaches its maximum intensity when $T_{2}$ is populated, and then, it lowers its intensity by a factor 5 when populating $\mathrm{T}_{1}$, to reach again a maximum for the subsequent population of $\mathrm{T}_{2}$.

This fact can also explain the reason for the experimental difficulties in assigning the eventual intermediate state of the proposed $S_{1} \rightarrow T_{2} \rightarrow T_{1}$ indirect mechanism: (i) the fast dynamical equilibrium between $T_{1}$ and $T_{2}$ (in most of the trajectories, they exchange in just a few fs) challenges available state-of-the-art ultrafast spectroscopies, and (ii) it is not possible to distinguish the different triplet states in the experimental spectrum because their signals overlap when averaging on the ensemble trajectories. We therefore conclude that the experimentally determined gas-phase characteristic time $(5.0 \pm 0.5 \mathrm{ps})$ should most probably be referred to the population of $\mathrm{T}_{2}$, in qualitative agreement with our parallel (theoretically oriented) kinetic model (3.38 ps) and in quantitative agreement with our serial (experimentally oriented) kinetic model (4.5 ps). A direct comparison with the experimental kinetic model is nevertheless not a straightforward task because the fitting procedure is experimentally based on the signal strength but theoretically based on the population.

From a vibrational point of view, after $200 \mathrm{fs}$, when the overall triplet population is already $20 \%, \mathrm{~T}_{1}$ and $\mathrm{T}_{2}$ populations lose their vibrational coherence. This is also suggested by the time-dependent oscillations of $d_{\mathrm{CO}}$ and $\Phi_{\text {plan }}$, which end up being highly scattered after an initial coherent (i.e., in-phase vibration) period (Figures S4 and S5 in the Supporting Information). 
On the other hand, the trajectories remaining in the initial $S_{1}$ state indicate that $S_{1} \rightarrow S_{0}$ fluorescence is, in principle, possible, even though it results in a marginal process, as evidenced by the subpicosecond $S_{1}$ lifetime $\left(\tau_{\mathrm{S} 1}=709\right.$ and $\left.683 \mathrm{fs}\right)$ obtained from our kinetic models (Figure 1). Indeed, a quasi-unitary ISC quantum yield $^{32}$ coupled to low fluorescence quantum yield ${ }^{20}$ was experimentally found. Moreover, the detected delayed fluorescence $^{20}$ can be explained in terms of recrossing events between singlet and triplet states $\left(S_{1} \rightarrow T_{1,2} \rightarrow S_{1}\right)$, as noticed in a small fraction $(5 \%)$ of our trajectories and predicted by the high SOC values calculated along the MEP. ${ }^{21}$

Both indirect and direct mechanisms for triplet population were recognized in our photodynamics study, spanned all over the simulation time (see Figures 2 and 3 and the Supporting
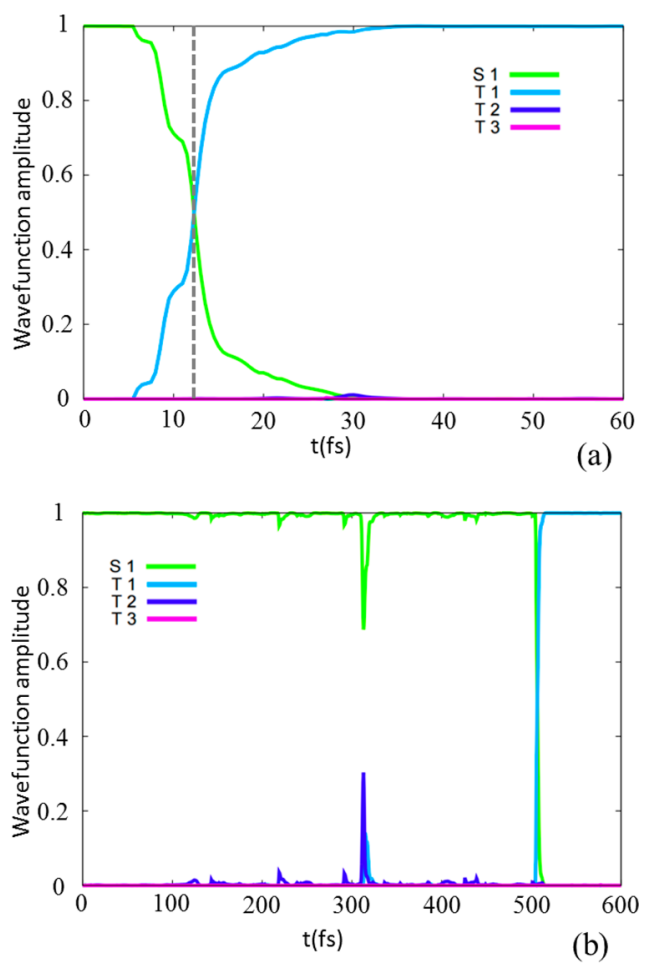

Figure 3. Representative excited-state trajectories showing an $S_{1} \rightarrow T_{1}$ direct mechanism at the first molecular vibration (a) or at a later stage (b).

Information). The indirect process was found to be prominent, as previously suggested. ${ }^{21}$ Nevertheless, a relevant percentage (33\% of the trajectories populating the triplet manifold) chose a direct process. Besides, even though the $T_{1}$ state is $\sim 10 \mathrm{kcal} /$ mol below $\mathrm{T}_{2}$ at the FC geometry, the SOC makes possible an $\mathrm{S}_{1} \rightarrow \mathrm{T}_{1}$ surface-hop already at the very first molecular vibration (Figure 3a), without requiring specific geometrical distortions. However, the direct process is also observed after several molecular vibrations (Figure $3 \mathrm{~b}$ ) because the energy gap is reduced during the dynamics, reaching a three-state $\left(S_{1}, T_{2}\right.$, and $T_{1}$ ) energy degeneracy, therefore generating a competition between $S_{1} \rightarrow \mathrm{T}_{2}$ (i.e., $\left.{ }^{1}\left(\mathrm{n}, \pi^{*}\right) \rightarrow{ }^{3}\left(\pi, \pi^{*}\right)\right)$ and $\mathrm{S}_{1} \rightarrow \mathrm{T}_{1}$ (i.e., $\left.{ }^{1}\left(\mathrm{n}, \pi^{*}\right) \rightarrow{ }^{3}\left(\mathrm{n}, \pi^{*}\right) /^{3}\left(\pi, \pi^{*}\right)\right)$ potential surface-hops along the dynamics.

In conclusion, we have shown by excited-state surfacehopping dynamics that BP can efficiently populate the triplet manifold by an ultrafast ISC process. The indirect mechanism $S_{1} \rightarrow T_{2} \rightarrow T_{1}$ is favored with respect to the direct $S_{1} \rightarrow T_{1}$ one (ratio 2:1). Nevertheless, in both cases, a quite persistent equilibrium between $T_{1}$ and $T_{2}$ is observed at $600 \mathrm{fs}$ (see Figure $\mathrm{S} 2 \mathrm{c}$ in the Supporting Information), indicating an unrelaxed triplet manifold not detailed before and in agreement with previous gas-phase experiments. ${ }^{5}$ This finding makes it possible to revisit the common spectroscopic assignment and allow both triplet channels for DNA photosensitization and photocatalysis to open, enhancing the feasibility of the energy-transfer process. Note that in solution, energy transfer to the solvent can be an additional factor to be considered. More in general, these results are relevant not only for $\mathrm{BP}$ but also for the other members of the diaryl ketones family in which the lowestenergy singlet excited state has $n \pi^{*}$ nature because no specific (i.e., system-dependent normal mode) molecular coordinate is explicitly involved in the process; this could be due to the extended $\left(S_{1}, T_{1}\right.$, and $\left.T_{2}\right)$ quasi-degeneracy and non-negligible SOCs between singlet and triplet PESs. Because of these findings, coupled to the mixed $n \pi^{*} / \pi \pi^{*}$ nature of the lowest triplet state, we consider that nonadiabatic dynamics play an important role in correctly determining the photochemical behavior of such systems.

\section{ASSOCIATED CONTENT}

\section{Supporting Information}

The Supporting Information is available free of charge on the ACS Publications website at DOI: 10.1021/acs.jpclett.5b02792.

Computational details, kinetic models parameters, singlepoint calculations of geometries along the potential energy surface, wavefunction amplitude graphs and geometrical analysis of the 39 trajectories populating the triplet manifold, Franck-Condon structure, and description of the transient absorption spectrum simulation (PDF)

\section{AUTHOR INFORMATION}

\section{Corresponding Authors}

*E-mail: marco.marazzi@univ-lorraine.fr (M.M.).

*E-mail: antonio.monari@univ-lorraine.fr (A.M.).

\section{Notes}

The authors declare no competing financial interest.

\section{ACKNOWLEDGMENTS}

This work has been supported by Université de Lorraine and French CNRS. M.M. is grateful to the French National Research Agency (ANR) for a postdoctoral contract in the framework of the $\mathrm{QMC}=\mathrm{Chem}$ project. D.R.-S. thanks the Spanish MINECO/FEDER (CTQ2014-58624-P project, JCI2012-13431 Juan de la Cierva fellowship, and Maria de Maetzu program/MDM-2015-0538) and the Generalitat Valenciana (GV2015-057) for financial support. S.M. and L.G. acknowledge the Austrian Science Fund (FWF) for financial support through Project P25827 as well as the COST action CM1405 (MOLIM).

\section{REFERENCES}

(1) Ohmori, N.; Suzuki, T.; Ito, M. Why Does Intersystem Crossing Occur in Isolated Molecules of Benzaldehyde, Acetophenone, and Benzophenone? J. Phys. Chem. 1988, 92, 1086-1093.

(2) Yabumoto, S.; Sato, S.; Hamaguchi, H. Vibrational and Electronic Infrared Absorption Spectra of Benzophenone in the Lowest Excited Triplet State. Chem. Phys. Lett. 2005, 416, 100-103. 
(3) El-Sayed, M. A. Low Field Zeeman Effect and the Mechanism of the S1 $\rightarrow$ T1 Nonradiative Process. J. Chem. Phys. 1975, 62, 1579.

(4) Batley, M.; Kearns, D. R. The Second Triplet State of Benzophenone. Chem. Phys. Lett. 1968, 2, 423-425.

(5) Matsushita, Y.; Kajii, Y.; Obi, K. Photochemical Reaction of Excited Benzophenone in the Gas Phase. J. Phys. Chem. 1992, 96, 4455-4458.

(6) Miyasaka, H.; Morita, K.; Kamada, K.; Mataga, N. FemtosecondPicosecond Laser Photolysis Studies on Photoreduction Process of Excited Benzophenone with N,N-Dimethylaniline in Acetonitrile Solution. Bull. Chem. Soc. Jpn. 1990, 63, 3385-3397.

(7) Aloïse, S.; Ruckebusch, C.; Blanchet, L.; Réhault, J.; Buntinx, G.; Huvenne, J.-P. The Benzophenone $\mathrm{S} 1\left(\mathrm{n}, \pi^{*}\right) \rightarrow \mathrm{T} 1\left(\mathrm{n}, \pi^{*}\right)$ States Intersystem Crossing Reinvestigated by Ultrafast Absorption Spectroscopy and Multivariate Curve Resolution. J. Phys. Chem. A 2008, 112, 224-231.

(8) Katoh, R.; Kotani, M.; Hirata, Y.; Okada, T. Triplet Exciton Formation in a Benzophenone Single Crystal Studied by Picosecond Time-Resolved Absorption Spectroscopy. Chem. Phys. Lett. 1997, 264, 631-635.

(9) Mitrić, R.; Petersen, J.; Bonačić-Koutecký, V. Laser-Field-Induced Surface-Hopping Method for the Simulation and Control of Ultrafast Photodynamics. Phys. Rev. A: At., Mol., Opt. Phys. 2009, 79, 053416.

(10) Richter, M.; Marquetand, P.; González-Vázquez, J.; Sola, I.; González, L. SHARC: Ab Initio Molecular Dynamics with Surface Hopping in the Adiabatic Representation Including Arbitrary Couplings. J. Chem. Theory Comput. 2011, 7, 1253-1258.

(11) Granucci, G.; Persico, M.; Spighi, G. Surface Hopping Trajectory Simulations with Spin-Orbit and Dynamical Couplings. J. Chem. Phys. 2012, 137, 22A501.

(12) Cui, G.; Thiel, W. Generalized Trajectory Surface-Hopping Method for Internal Conversion and Intersystem Crossing. J. Chem. Phys. 2014, 141, 124101.

(13) Mai, S.; Richter, M.; Ruckenbauer, M.; Oppel, M.; Marquetand, P.; González, L. SHARC: Surface Hopping Including Arbitrary Couplings - Program Package for Non-Adiabatic Dynamics. http://sharc-md.org/ (2014).

(14) Carroll, G. T.; Turro, N. J.; Koberstein, J. T. Patterning Dewetting in Thin Polymer Films by Spatially Directed Photocrosslinking. J. Colloid Interface Sci. 2010, 351, 556-560.

(15) Ibrahim, N. A.; El-Zairy, E. M. R.; Abdalla, W. A.; Khalil, H. M. Combined UV-Protecting and Reactive Printing of Cellulosic/wool Blends. Carbohydr. Polym. 2013, 92, 1386-1394.

(16) Cuderman, P.; Heath, E. Determination of UV Filters and Antimicrobial Agents in Environmental Water Samples. Anal. Bioanal. Chem. 2007, 387, 1343-1350.

(17) Dorman, G.; Prestwich, G. D. Benzophenone Photophores in Biochemistry. Biochemistry 1994, 33, 5661-5673.

(18) Dumont, E.; Monari, A. Benzophenone and DNA: Evidence for a Double Insertion Mode and Its Spectral Signature. J. Phys. Chem. Lett. 2013, 4, 4119-4124.

(19) Dumont, E.; Wibowo, M.; Roca-Sanjuán, D.; Garavelli, M.; Assfeld, X.; Monari, A. Resolving the Benzophenone DNA-Photosensitization Mechanism at QM/MM Level. J. Phys. Chem. Lett. 2015, $6,576-580$.

(20) Brown, R. E.; Singer, L. A.; Parks, J. H. Prompt and Delayed Fluorescence from Benzophenone. Chem. Phys. Lett. 1972, 14, 193195.

(21) Sergentu, D.-C.; Maurice, R.; Havenith, R. W. a.; Broer, R.; Roca-Sanjuán, D. Computational Determination of the Dominant Triplet Population Mechanism in Photoexcited Benzophenone. Phys. Chem. Chem. Phys. 2014, 16, 25393-25403.

(22) Tully, J. C. Molecular Dynamics with Electronic Transitions. J. Chem. Phys. 1990, 93, 1061-1071.

(23) Tomasi, J.; Mennucci, B.; Cammi, R. Quantum Mechanical Continuum Solvation Models. Chem. Rev. 2005, 105, 2999-3093.

(24) El-Sayed, M. A. Triplet State. Its Radiative and Nonradiative Properties. Acc. Chem. Res. 1968, 1, 8-16.
(25) Spighi, G.; Gaveau, M.-A.; Mestdagh, J.-M.; Poisson, L.; Soep, B. Gas Phase Dynamics of Triplet Formation in Benzophenone. Phys. Chem. Chem. Phys. 2014, 16, 9610-9618.

(26) Aquilante, F.; Autschbach, J.; Carlson, R. K.; Chibotaru, L. F.; Delcey, M. G.; De Vico, L.; Fdez Galván, I.; Ferré, N.; Frutos, L. M.; Gagliardi, L.; et al. Molcas 8: New Capabilities for Multiconfigurational Quantum Chemical Calculations across the Periodic Table. J. Comput. Chem. 2016, 37, 506.

(27) Boström, J.; Delcey, M. G.; Aquilante, F.; Serrano-Andrés, L.; Pedersen, T. B.; Lindh, R. Calibration of Cholesky Auxiliary Basis Sets for Multiconfigurational Perturbation Theory Calculations of Excitation Energies. J. Chem. Theory Comput. 2010, 6, 747-754.

(28) Delcey, M. G.; Freitag, L.; Pedersen, T. B.; Aquilante, F.; Lindh, R.; González, L. Analytical Gradients of Complete Active Space SelfConsistent Field Energies Using Cholesky Decomposition: Geometry Optimization and Spin-State Energetics of a Ruthenium Nitrosyl Complex. J. Chem. Phys. 2014, 140, 174103.

(29) Minns, R. S.; Parker, D. S. N.; Penfold, T. J.; Worth, G. A.; Fielding, H. H. Competing Ultrafast Intersystem Crossing and Internal Conversion in the "Channel 3" Region of Benzene. Phys. Chem. Chem. Phys. 2010, 12, 15607-15615.

(30) Ramseier, M.; Senn, P.; Wirz, J. Photohydration of Benzophenone in Aqueous Acid. J. Phys. Chem. A 2003, 107, 33053315.

(31) Petit, A.; Subotnik, J. E. Calculating Time-Resolved Differential Absorbance Spectra for Ultrafast Pump-Probe Experiments with Surface Hopping Trajectories. J. Chem. Phys. 2014, 141, 154108.

(32) Terazima, M.; Hirota, N. Measurement of the Quantum Yield of Triplet Formation and Short Triplet Lifetimes by the Transient Grating Technique. J. Chem. Phys. 1991, 95, 6490-6495. 